\title{
DIAGNOSTIC DILEMMA IN A CASE OF BILATERAL TESTICULAR SWELLINGS: A CASE REPORT
}

Santanu Sarkar ${ }^{1}$, Sukhamoy Saha ${ }^{2}$, Manabendra Naskar ${ }^{3}$, Madhusudhan Saha4, Prasenjeet Mondal ${ }^{5}$

\section{HOW TO CITE THIS ARTICLE:}

Santanu Sarkar, Sukhamoy Saha, Manabendra Naskar, Madhusudhan Saha, Prasenjeet Mondal. "Diagnostic Dilemma in a case of Bilateral Testicular Swellings: A Case Report". Journal of Evolution of Medical and Dental Sciences 2014; Vol. 3, Issue 29, July 21; Page: 8086-8089, DOI: 10.14260/jemds/2014/3016

ABSTRACT: Isolated tubercular epididymo-orchitis usually presents with a clinical pictures and radiographic illustrations similar to those of a testicular neoplasm; therefore diagnosis may be challenging for clinicians. In our case, a 25years male presented with bilateral testicular swellings without any evidence of tuberculosis elsewhere in the body. We report this case firstly because isolated epididymo-orchitis is an unusual presentation of tuberculosis. Secondly this case may help clinicians in diagnostic approach when testicular tuberculosis masquerade testicular neoplasm, and thus avoids unnecessary orchiectomy and save the clinicians from the possible charges of medical negligence.

KEYWORDS: Diagnostic Dilemma; Tubercular epididymo-orchitis; Testicular neoplasms; High resolution ultrasonography.

INTRODUCTION: Genitourinary tuberculosis is the $2^{\text {nd }}$ most common manifestations of extra pulmonary tuberculosis next to tubercular-lymphadenopathy and genitourinary involvement has been noted in $7 \%$ patients with tuberculosis at autopsy. The prostate, epididymis and seminal vesicles are the most commonly affected organs, while testicular involvement is very rare.[1,2]

Extra-genital involvement including pulmonary and renal tuberculosis can be documented in $50 \%$ and $80-85 \%$ respectively in patients with genital tuberculosis.[3] Our case demonstrates unusual presentation of genital tuberculosis without any evidence of pulmonary or renal involvement.

As most of the cases of tubercular epididymo-orchitis presented with similar clinical features of testicular neoplasm; unfortunately, still today most of the patients are still undergone orchiectomy. On the other hand if testicular tuberculosis left untreated it has considerable deleterious effect on fertility. The sperm counts and motility may be reduced due to blockage of the vas or secondary atrophy ${ }^{[4]}$ The presence of abscess or sinus formation indicates advanced scrotal disease.

CASE REPORT: A 25 years male, non-smoker presented in surgery OPD with complains of swelling of both testes with pain over a period of 6 months; Testicular swelling was gradually increasing in size. There was no history of fever, weight loss, night sweats, cough or haemoptysis. Even there were no history of dysuria, frequency, urgency of micturition or history of any trauma. He has two children. clinical examination of chest and the abdomen were normal.

Physical examination of the genitalia revealed firm enlargement of both testes with irregular surface, while epididymis and spermatic cords were thickened. The scrotal skin showed no signs of inflammation. Transillumination test of the swellings was negative. There was bilateral inguinal lymphadenopathy. Prostate was found normal on digital rectal examination. 
His Complete blood count, biochemistry tests and serum tumour markers including alphafetoprotein, beta-human chorionic gonadotropin and LDH were all within normal limits. Urinalysis was normal including urine culture. VDRL and HIV tests were negative. Chest X-ray was normal. Ultrasonography (USG) of abdomen showed retroperitoneal lymphadenopathy. Scrotal high resolution ultrasound showed gross enlargement with evidence of multiple small hypoechoic areas along with focal areas of calcifications in both testes; these findings were consistent with chronic infective granuloma. Fine needle aspiration cytology of inguinal lymph nodes showed reactive hyperplasia.

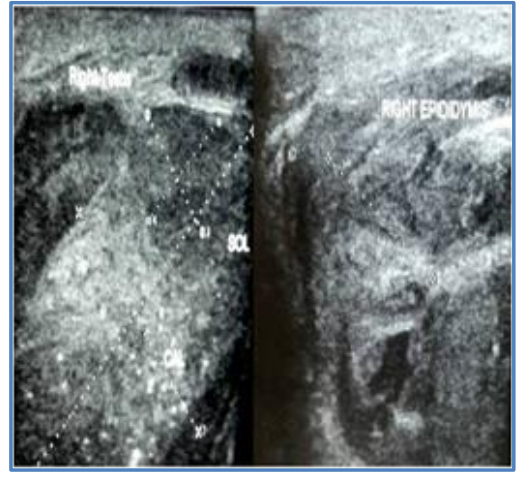

Figure 1 (a)

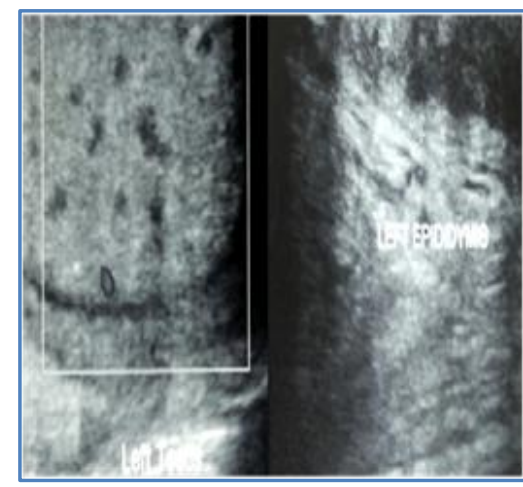

Figure 1 (b)

Figure 1: 1(a) USG shows multiple hypoechoic areas and prominent calcifications in Rt. testis with heterogeneously thickened Rt. epididymis. 1(b) USG of Lt. testis shows multiple hypoechoic areas and few calcifications and also heterogeneously thickened Lt. epididymis

We initially suspected of bilateral testicular neoplasm probably germ cell tumour from histoty, clinical examination and retroperitoneal lymphadenopathy on abdominal ultrasound. But as ultrasound picture of both testes were highly suggestive of tuberculosis we planned for bilateral testicular and epididymal biopsy instead of orchidectomy. Histopathological examination was consistent with tubercular epididymo-orchitis [Figure 2(a), 2(b)]. He received category-1 antitubercular treatment with isoniazid, rifampicin, ethambutol and pyrazinamide and 6months later no sign of the disease was noticed either clinically or radiologically. The patient is under follow-up for last 6 months.

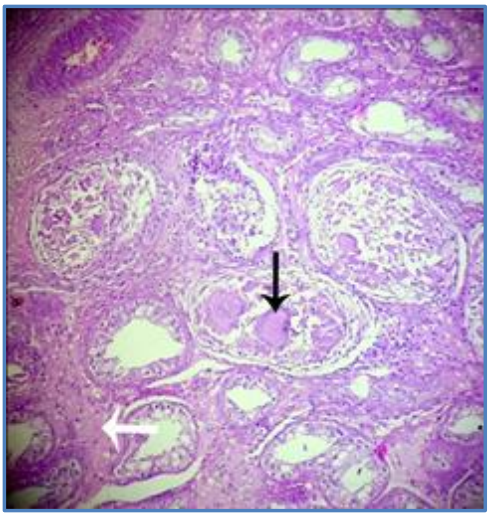

Figure 2 (a)

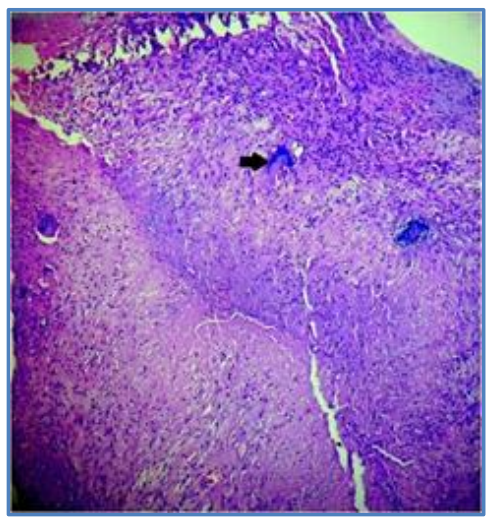

Figure 2 (b) 
Figure 2: 2 (a) Low-power view of right testis showing multiple Langhan's giant cells(black arrow) involving seminiferous tubules and caseous necrosis(white arrow) [H\&E; $\times 100$ ].

2(b) Low power view of left epididymis showing effacement of normal structure by granulomas and Langhan's giant cells (black arrow) [H \& E; ×100].

DISCUSSION: Genital tuberculosis is usually a disease of sexually active males, most commonly occurs between the ages of 20 to 40 years. The spread of tuberculosis to the epididymis is either haematogenously or by retrocanalicular descent of organisms from the haematogenously infected prostate. Distal spread through the genitourinary tract from a renal source also possible. Testicular involvement is usually as a result of local invasion from the epididymis, retrograde seeding from the epididymis or rarely by haematogenous spread.[5]

High resolution sonography is currently the best technique for imaging the scrotum and its contents. ${ }^{[6]}$ Diagnosis of tubercular epididymo-orchitis confirmed by histopathological examination. In addition to biopsy/FNAC a polymerase chain reaction (PCR) when available may result in rapid detection of Mycobacterium tuberculosis. ${ }^{[7]}$ Multidrug chemotherapy is the treatment of choice for tubercular epididymo-orchitis; surgery is indicated in unresponsive cases only.

CONCLUSION: Testicular biopsy is contraindicated in suspected case of malignancy and on the other hand diagnosis of tubercular epididymo-orchitis can't be achieved without histopathological examination. Our case reestablished the utility of good quality ultrasonography when there is dilemma in clinician's mind for proper diagnostic approach for such cases. A high index of suspicion is also necessary especially in endemic areas and thus avoids unnecessary orchiectomy in a potentially curable medical disease in a sexually active male, and save the clinicians from the possible charges of medical negligence.

\section{REFERENCES:}

1. Rosenberg S. Has chemotherapy reduced the incidence of genitourinary tuberculosis: A comparison based on necropsy material from Bellevue Hospital. J Urol 1963; 90: 317.

2. Hetal Shah, Keyur Shah, Ramakant Dixit, Kusum V. Shah. Isolated Tuberculous EpididymoOrchitis. Indian J Tuberc 2004; 51: 159-62.

3. Biswas M, Rahi R, Tiwary SK, Khanna R, Khanna AK. Kathmandu University Medical Journal 2006; 4: 98-99.

4. Gorse GJ, Belshe RB. Male genital tuberculosis: a review of the literature with instructive case reports. Rev Infect Dis 1985; 7: 511-2.

5. Misra MC, Kapoor VK, Mukhopadhyay AK, Sharma L. K. Tuberculosis of testis-an unusual presentation. Indian J Tuberc 1985; 32:160-161.

6. Muttarak M, Peh WCG, Lojanapiwat B, Chaiwun B. Tuberculous epididymitis and epididymoorchitis: sonographic appearances. Am J Roentgenol 2001; 176:1459-1466.

7. Wise GJ, Shteynshlyuger A. An update on lower urinary tract tuberculosis. Curr Urol Rep 2008; 9: 305-313. 


\section{CASE REPORT}

\section{AUTHORS:}

1. Santanu Sarkar

2. Sukhamoy Saha

3. Manabendra Naskar

4. Madhusudhan Saha

5. Prasenjeet Mondal

\section{PARTICULARS OF CONTRIBUTORS:}

1. Clinical Tutor in General Surgery, Department of General Surgery, Burdwan Medical College \& Hospital, Burdwan, West Bengal, India.

2. Assistant Professor in General Surgery, Department of General Surgery, Burdwan Medical College \& Hospital, Burdwan, West Bengal, India.

3. PGT in General Surgery, Department of General Surgery, Burdwan Medical College \& Hospital, Burdwan, West Bengal, India.
4. PGT in General Surgery, Department of General Surgery, Burdwan Medical College \& Hospital, Burdwan, West Bengal, India.

5. PGT in General Surgery, Department of General Surgery, Burdwan Medical College \& Hospital, Burdwan, West Bengal, India.

\section{NAME ADDRESS EMAIL ID OF THE} CORRESPONDING AUTHOR:

Santanu Sarkar,

\#1/3/1C

Ramlal Agarwala Lane,

Sinthee, Kolkata-700050.

Email: drsantanu.surg@gmail.com

Date of Submission: 03/07/2014.

Date of Peer Review: 04/07/2014.

Date of Acceptance: 14/07/2014.

Date of Publishing: 17/07/2014. 